\title{
Research on the Relationship between CPI and PPI Based on VEC Model
}

\author{
Shijun Li, Guoqiang Tang*, Duancui Yang*, Shixue Du \\ School of Science, Guilin University of Technology, Guilin, China \\ Email: *tanggq@glut.edu.cn, *ydc123@glite.edu.cn
}

How to cite this paper: Li, S.J., Tang, G.Q., Yang, D.C. and Du, S.X. (2019) Research on the Relationship between CPI and PPI Based on VEC Model. Open Journal of Statistics, 9, 218-229.

https://doi.org/10.4236/ojs.2019.92016

Received: March 14, 2019

Accepted: April 9, 2019

Published: April 12, 2019

Copyright ( 2019 by author(s) and Scientific Research Publishing Inc. This work is licensed under the Creative Commons Attribution International License (CC BY 4.0).

http://creativecommons.org/licenses/by/4.0/

(c) (i) Open Access

\begin{abstract}
By establishing the VEC model, the relationship between Consumer Price Index (CPI) and Producer Price Index (PPI) is explored by using Johansen cointegration test and impulse response function. The results show that there is a long-term equilibrium cointegration relationship between CPI and PPI. CPI has a certain impact on PPI. PPI also has a certain impact on CPI. PPI has a great impact on itself both in the long-term and short-term. The current CPI will be adversely affected by the previous CPI and the positive impact of the previous PPI. The current PPI will be positively affected by the previous phase of CPI and the previous phase of PPI.
\end{abstract}

\section{Keywords}

VEC Model, Consumer Price Index, Producer Price Index, Impulse Response Function

\section{Introduction}

The degree of inflation is mainly measured by the price index. The most important indicator is the Consumer Price Index (CPI), and Producer Price Index (PPI) can help determine the status quo and trends of inflation. CPI mainly reflects the price changes of daily life commodities and service items of urban households. It is one of the important indexes for judging the degree and trend of inflation. CPI is divided into food, tobacco, alcohol and supplies, household equipment and maintenance services, health care and personal goods, transportation and communications, entertainment, education, cultural goods and services, and housing. PPI can be regarded as the most important price index of the mid-stream industry, and it plays a role in the product price trend. PPI represents the production materials and consumer goods sold by industrial enterprises to commercial, foreign trade, materials, residents or other industries 
and sectors. It reflects the impact of ex-factory price changes on industrial output value within a certain period of time. It is not only the main indicator for judging inflation, but also the important basis for the formulation of policies PPI covers more than 4000 products in 39 industrial sectors. The National Bureau of Statistics divides the PPI into production data PPI and living data PPI. The production data PPI can be subdivided into extractive industry production data PPI, raw material production data PPI and processing industry production data PPI. And living data PPI can be subdivided into food products PPI, clothing PPI, general merchandise PPI and consumer durables PPI. Economic life can be understood depth by studying the relationship between CPI and PPI [1].

Through the price conduction law, the fluctuation of PPI will be transmitted to CPI. In turn, the fluctuation of CPI will also affect PPI. The two price indices can be described as an industrial chain connecting from upstream production to downstream consumption. PPI reflects upstream. With changes in production prices, CPI reflects changes in downstream consumption. According to the theory of production chain transfer, from the perspective of supply shock, price fluctuations are generally transmitted from upstream to downstream. Eventually, the fluctuation of PPI will cause CPI changes, but there will be a certain lag period; demand decision theory holds that the ultimate goal of all economic activities is to provide goods and services to consumers, so the consumer's demand will determine the price of these final consumer goods, and ultimately reflect the reverse mechanism of CPI to PPI. More research shows that the causal relationship between CPI and PPI is not one-way, but two-way, complex and non-linear [2]. The difference in CPI-PPI reflects the growth of corporate earnings: CPI-PPI has a strong leading role in industrial enterprise profits. The CPI-PPI indicator also has a strong correlation with the overall growth rate of the economy. The smaller the difference of CPI-PPI, the higher the operating cost of the enterprise, the lower the profit of the enterprise, the slower growth of the profit of the enterprise, and the slower economic growth. The greater the difference between CPI and PPI, the more the corporate profits are rebounding. The growth rate of corporate profits is rising, and the economic growth momentum is strong.

Many scholars have studied the relationship between CPI and PPI. Chen Yu (2011) studied the relationship between PPI, enterprise commodity price index, M2 and CPI through the Granger causality test and cointegration theory, and indirectly confirmed that PPI is one-way causality of CPI [3]. He Liping and Fan Gang and other scholars (2008) used the CPI and PPI data from January 2001 to July 2008 to conduct empirical research. The Granger causality test showed that there was One-way transmission CPI to PPI. To the conduction mechanism, the lag of conduction time is about 1 - 3 months [4]; Xu Weikang (2010) used the same data as the He Liping to find the Granger reason for each other in the short and long term [5]. Yang Zihui (2013) and Yang Can (2013) used empirical research on nonlinear Granger causality test and non-parametric frequency domain method. The conclusion is that CPI and PPI are complex nonlinear causality [6] [7]. 
Previous studies have shown that there is a certain relationship between CPI and PPI, and both are important indexes reflecting the trend of economic development. Therefore, this paper studies the relationship between CPI and PPI by fitting the VEC model, and in-depth study of its internal relationship will help to understand the internal law of macroeconomic operation and facilitate the formulation and implementation of policies. The structure of the article is as follows: Section 1 is the introduction; Section 2 is the model introduction and modeling steps; Section 3 is the empirical study; Section 4 is the conclusion.

\section{Model Introduction and Modeling Steps}

\subsection{VAR Model}

The vector autoregressive model (VAR model) was proposed by Sims C A in 1980 and is mainly used to analyze and predict the dynamic impact of random disturbance on the system, the magnitude of the impact, the positive and negative and the duration of time [8]. The focus of the VAR model is not on interpreting the estimated parameters, but on interpreting and analyzing the interrelationships among the variables through the impulse response function and the variance decomposition.

Let $Y_{t}=\left(y_{1 t}, y_{2 t}, \cdots, y_{n t}\right)^{\prime}$ denote an $(n \times 1)$ vector of time series variables. The basic p-lag vector autoregressive $(\operatorname{VAR}(p))$ model has the form:

$$
Y_{t}=c+\prod_{1} y_{t-1}+\prod_{2} y_{t-2}+\cdots+\prod_{p} y_{t-p}+\varepsilon_{t}, \quad t=1,2, \cdots, T
$$

where $\prod_{i}$ are $(n \times n)$ coefficient matrices and $\varepsilon_{t}$ is an $(n \times 1)$ unobservable zero mean white noise vector process (serially uncorrelated or independent) with time invariant covariance matrix $\sum$. The stability condition of the $\operatorname{VAR}(p)$ model is that the characteristic equation is required. $\left|\Pi_{i}-\lambda \mathrm{E}\right|=0$ The roots are all within the unit circle.

\subsection{Johansen Cointegration Test [9]}

In 1990, Johansen and Juselius proposed the Johansen cointegration test, which is a test method for testing regression coefficients based on the VAR model, and can also be used for cointegration tests between multiple variables.

For $Y_{t}=\left(y_{1 t}, y_{2 t}, \cdots, y_{n t}\right)^{T}$, a cointegration definition can be used to discuss whether there is a cointegration relationship between this variable. The definition of cointegration is as follows:

The components of the n-dimensional vector time series $Y_{t}$ are called $d$, $b$-order cointegration. Recorded as $Y_{t} \sim C I(d, b)$, if the following two points are met:

1) $Y_{t} \sim I(d)$, require that each component of $Y_{t}$ is $d$-order and monolithic;

2) There is a non-zero vector $\beta$, make $\beta^{\prime} Y_{t} \sim I(d-b), 0<b \leq d$.

The abbreviation $Y_{t}$ is cointegration, and the vector $\beta$ becomes a cointegration vector.

There are at most $n-1$ linearly independent cointegration vectors for the $n$-dimensional vector time series $Y_{t}$. 


\subsection{The Basic Idea of Impulse Response Function [10]}

In practical applications, because the VAR model is a non-theoretical model, it does not have to make any a priori constraints on the variables, so when analyzing the VAR model, it is generally not analyzed that one variable will produce another variable. How to influence, but to analyze the dynamic impact on the system when an error term changes, that is, when the model is subjected to some kind of impact, this analysis method is called the impulse response function.

The impulse response function analysis method can be used to describe the response of the impact of the error term on the endogenous variable, that is, the impact of the impact of one standard deviation on the random error term on the current value and future value of the endogenous variable.

The basic idea of the impulse response function analysis method is introduced below by taking the $\operatorname{VAR}(2)$ model with two variables as an example.

$$
\left\{\begin{array}{l}
y_{1 t}=a_{1} y_{1 t-1}+a_{2} y_{1 t-2}+b_{1} y_{2 t-1}+b_{2} y_{2 t-2}+\varepsilon_{1 t} \\
y_{2 t}=c_{1} y_{1 t-1}+c_{2} y_{1 t-2}+d_{1} y_{2 t-1}+d_{2} y_{2 t-2}+\varepsilon_{2 t}
\end{array}, t=1,2, \cdots, T\right.
$$

In formula (2), Pending parameter $a_{i}, b_{i}, c_{i}, d_{i}, \varepsilon_{t}=\left(\varepsilon_{1 t}, \varepsilon_{2 t}\right)^{\prime}$ random disturbance. Assume that it is a white noise vector with the following properties:

$$
\begin{aligned}
& \mathrm{E}\left(\varepsilon_{i t}\right)=0, \forall t, i=1,2 \\
& \operatorname{var}\left(\varepsilon_{t}\right)=\mathrm{E}\left(\varepsilon_{t} \varepsilon_{t}^{\prime}\right)=\sum=\left\{\sigma_{i j}\right\}, \forall t \\
& \mathrm{E}\left(\varepsilon_{i t} \varepsilon_{i s}\right)=0, \forall t \neq s, i=1,2
\end{aligned}
$$

Assume that it is starting from the 0th period, and set

$$
y_{1 t-1}=y_{1 t-2}=y_{2 t-1}=y_{2 t-2}=0 \text {, }
$$

Also set in the 0 th period given the disturbance term $\varepsilon_{10}=1, \varepsilon_{20}=0$, And other disturbance items are $0 . \varepsilon_{1 t}=\varepsilon_{2 t}=0(t=1,2, \cdots)$. This is called the impulse response of phase 0 to $y_{1 t}$.

\subsection{VEC Model [10]}

Engle and Granger combine the cointegration theory with the error correction model to establish a vector error correction model, the VEC model. As long as there is a cointegration relationship between the variables, the error correction model (ECM) can be derived from the autoregressive distribution lag model. In the VAR model, each equation is an autoregressive distribution lag model. Therefore, we can think that the VEC model is a VAR model with cointegration constraints.

According to the cointegration equation, the expression of the VEC model can be obtained:

$$
\Delta y_{t}=\alpha \beta^{\prime} y_{t-1}+\sum_{i=1}^{p-1} \Gamma_{i} \Delta y_{t-i}+\mu_{t}
$$

among them, $\Gamma_{i}=-\sum_{j=i+1}^{p} \Pi_{j}, \Delta y_{t}$ is the stationary value of $y_{t}$ after differential transformation, $\alpha$ is a coefficient vector, $\beta$ is a cointegration vector, and $\mu_{t}$ is a random perturbation vector. 
Equation (4) can also be expressed as:

$$
\Delta y_{t}=\alpha \cdot \mathrm{ecm}_{t-1}+\sum_{i=1}^{p-1} \Gamma_{i} \Delta y_{t-i}+\mu_{t}
$$

Each equation in Equation (5) is an error correction model. Where $\mathrm{ecm}_{t-1}=\beta^{\prime} y_{t-1}$ is the error correction term vector, reflecting the long-term equilibrium relationship between variables. The coefficient vector $\alpha$ reflects the adjustment speed of the variable to the equilibrium state when it deviates from the long-term equilibrium state. All coefficients of the difference term as explanatory variables reflect the effect of short-term fluctuations of each variable on short-term changes as explanatory variables.

\subsection{Modeling Steps}

This paper explores the relationship between CPI and PPI by establishing a VEC model and using co-integration test and impulse response function. The modeling steps are as follows:

1) In order to eliminate seasonal trends and heteroscedasticity and reduce fluctuations, first-order differences between CPI sequences and PPI sequences to conduct DCPI and DPPI sequences;

2) Perform unit root test on sequence CPI, PPI, DCPI, DPPI;

3) Determining the maximum lag order $\mathrm{p}$ of the VAR model by the SC criterion;

4) Construct the $\operatorname{VAR}(p)$ model with the maximum lag order $p$, and verify the stability of the $\operatorname{VAR}(p)$ model using the AR root chart;

5) Johansen cointegration test on the first-order single-sequence CPI and PPI, and check whether there is a long-term cointegration relationship;

6) Based on the cointegration test, the impulse response function of the VAR model is analyzed;

7) Explore the short-term fluctuations and long-term equilibrium between CPI and PPI through the VEC model.

\section{Empirical Research}

\subsection{Data Source}

The data in this paper comes from the Eastern Fortune Network. The monthly data of China's consumer price index (CPI) and industrial producers' price index (PPI) from January 2008 to December 2018 are selected. The total data are 132 months. Figure 1 shows time series chart of the Consumer Price Index (CPI) and the Producer Price Index (PPI). It can be seen from Figure 1 that the PPI is always higher than CPI from November 2016 to November 2018. PPI fluctuates sharply compared to CPI.

\subsection{Sequence Stability Test}

It can be seen from Figure 1 that the CPI sequence and the PPI sequence change with time. The time serieschart shows that the CPI sequence and the PPI sequence have obvious non-stationarity. The volatility of the PPI sequence is 


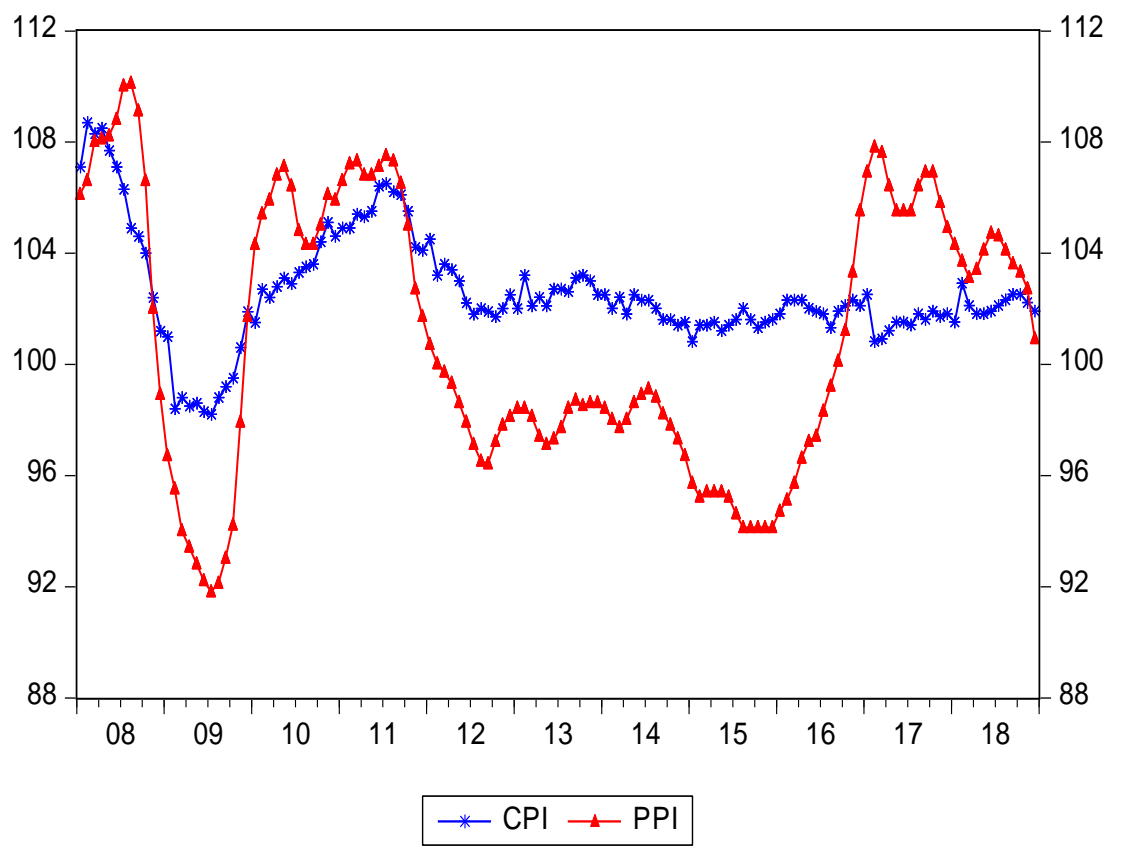

Figure 1. Time series diagram of CPI and PPI.

obviously stronger than that CPI sequence. From the objective point of view, it is necessary to judge the stationarity of the sequence by unit root test. In order to eliminate seasonal trends and heteroscedasticity and reduce fluctuations, the sequence CPI and PPI are subjected to first-order difference respectively, and the generated sequences are recorded as sequence DCPI and DPPI respectively, and the unit root test are performed on the two sequences. The $P$-value is defined as the probability, under the null hypothesis $H, \operatorname{Pr}(X \geq x \mid H)$ for right tail event, $\operatorname{Pr}(X \leq x \mid H)$ for left tail event, $2 \min \{\operatorname{Pr}(X \leq x \mid H), \operatorname{Pr}(X \geq x \mid H)\}$ for double tail event.

It can be seen from Table 1 that the ADF test values of the sequence CPI and PPI are greater than the $t$-statistic thresholds of the test levels of $1 \%, 5 \%$, and $10 \%$, and the probability $P$ values are all greater than 0.10 . The original hypothesis cannot be rejected, the sequence CPI and PPI are non-stationary sequence. The differenced sequence is DCPI and DPPI. The ADF test value is less than the $t$-statistic threshold of $1 \%, 5 \%$ and $10 \%$ of the test level, and the probability $P$ value is less than 0.01 , which means the sequence DCPI and DPPI are considered to be stationary sequences. The sequence CPI and PPI are first order single-order sequences, indicating that the two may have a long-term co-integration relationship.

\subsection{VAR Model Maximum Lag Order}

Before constructing the VAR model, the Schwartz Criterion (SC) was used to determine the maximum lag order $\mathrm{p}$ of the model. The results are shown in Table 2.

It can be seen from Table 2 that the minimum of order $P$ corresponding to 
Table 1. Unit root test results of two sequences.

\begin{tabular}{ccccccc}
\hline \multirow{2}{*}{ sequence } & ADF test value & \multicolumn{3}{c}{ Significant level } & \multirow{2}{*}{$P$ Pvalue } & Stationarity \\
\cline { 3 - 6 } & & $1 \%$ & $5 \%$ & $10 \%$ & & \\
\hline CPI & 0.066846 & -2.584539 & -1.943540 & -1.614941 & 0.7022 & Non-stable \\
PPI & -0.707841 & -2.583011 & -1.943324 & -1.615075 & 0.4084 & Non-stable \\
DCPI & -6.427866 & -2.584539 & -1.943540 & -1.614941 & 0.0000 & smooth \\
DPPI & -4.920177 & -2.583011 & -1.943324 & -1.615075 & 0.0000 & smooth \\
\hline
\end{tabular}

Table 2. Maximum lag order selection criteria.

\begin{tabular}{ccccccc}
\hline Lag & LogL & LR & FPE & AIC & SC & HQ \\
\hline 0 & -571.0934 & NA & 35.43458 & 9.243442 & 9.288930 & 9.261920 \\
1 & -271.4000 & 584.8855 & 0.300730 & 4.474193 & 4.610658 & 4.529628 \\
2 & -191.6153 & 153.1351 & 0.088584 & 3.251859 & $3.479301^{*}$ & 3.344252 \\
3 & -183.7764 & 14.79282 & 0.083278 & 3.189941 & 3.508360 & $3.319290^{*}$ \\
4 & -177.6029 & $11.45070^{*}$ & $0.080431^{*}$ & $3.154886^{*}$ & 3.564282 & 3.321192 \\
5 & -175.3695 & 4.070517 & 0.082791 & 3.183380 & 3.683752 & 3.386643 \\
6 & -175.2173 & 0.272599 & 0.088145 & 3.245440 & 3.836789 & 3.485660 \\
7 & -174.1344 & 1.903796 & 0.092469 & 3.292490 & 3.974816 & 3.569667 \\
8 & -169.2561 & 8.419016 & 0.091268 & 3.278324 & 4.051627 & 3.592458 \\
\hline
\end{tabular}

the SC is 2, so the VAR model selects the lag order $P=2$ as the maximum lag order.

\subsection{Establishment and Verification of VAR Model}

To establish a matrix form of the VAR(2) model:

$$
\begin{aligned}
\left(\begin{array}{l}
\mathrm{CPI} \\
\mathrm{PPI}
\end{array}\right)_{t}= & \left(\begin{array}{l}
8.916056 \\
3.592720
\end{array}\right)+\left(\begin{array}{ll}
0.768439 & 0.219738 \\
0.180074 & 1.743875
\end{array}\right)\left(\begin{array}{l}
\mathrm{CPI} \\
\mathrm{PPI}
\end{array}\right)_{t-1} \\
& +\left(\begin{array}{cc}
0.138832 & -0.214378 \\
-0.169544 & -0.790326
\end{array}\right)\left(\begin{array}{l}
\mathrm{CPI} \\
\mathrm{PPI}
\end{array}\right)_{t-2}+\left(\begin{array}{l}
\mu_{1 t} \\
\mu_{2 t}
\end{array}\right)
\end{aligned}
$$

The SC value of the VAR(2) model is 3.479301, and the SC value is the smallest, indicating that the established $\operatorname{VAR}(2)$ model works well. In order to verify the smoothness of the VAR model, the AR root chart is used for verification. It can be seen from Figure 2 that the modulus of all unit root reciprocals falls within the unit circle, which means the established VAR(2) model is stable. The $X$ and $Y$ axes of Figure 2 represent the coefficients of the eigenvalues, respectively. The four points in the unit circle are $(0.92,0.00),(0.89,-0.18),(0.89$, $0.18),(-0.19,0.00)$.

\subsection{Johansen Cointegration Test}

When the sequence is a non-stationary, pseudo-regression may occur in the 
Inverse Roots of AR Characteristic Polynomia

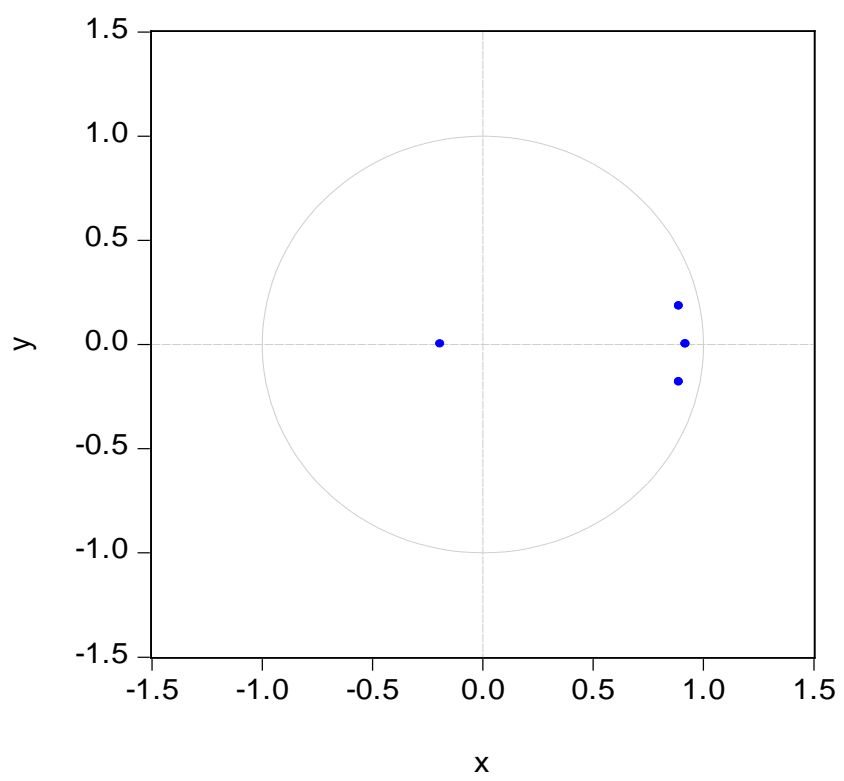

Figure 2. Distribution of AR unit roots.

construction model. The purpose of the cointegration test is to test whether the causal relationship described by the regression equation is a pseudo-regression. That is to say, whether there is a long-term stable relationship between the variables. The cointegration test requires that each sequence must be a non-stationary sequence and single-order single-sequence. From the unit root test results, it can be known that the sequence CPI and PPI meet the requirements of the cointegration test.

It can be seen from Table 3 that at the $5 \%$ significance level, the trace statistic test value is greater than the critical value, and the $\mathrm{p}$ values of the first row and the second row are 0.0007 and 0.0025 , both are less than 0.05 , rejecting the null hypothesis that there's no cointegration relationship. The conclusion is that there is a long-term cointegration relationship between the consumer price index and the industrial producer's ex-factory price index. After standardizing the cointegration coefficient, the resulting cointegration equation is as follows:

$$
\mathrm{CPI}=0.923276 \mathrm{PPI}+\mu_{t}
$$

\subsection{Analysis of Impulse Response Function}

In order to describe the impact of a standard deviation on the random error term on the current and future values of the variable, this paper uses the impulse response function to analyze the response of consumer price index to the producer price index.

From the impulse response results in Figure 3, the impact of consumer price index on itself, reached a maximum of 0.53 at the first period, then began to decline to 0.44 of the second period, then rose to 0.46 in the third period, and then began a slow decline. It can be seen that consumer price index has short-term 
Table 3. Johansen cointegration test results.

\begin{tabular}{ccccc}
\hline $\begin{array}{c}\text { Null hypothesis: } \\
\text { Number of cointegration vectors }\end{array}$ & Eigenvalues & Trace statistics & $\begin{array}{c}0.05 \text { significance } \\
\text { level }\end{array}$ & $P$ value \\
\hline None $^{*}$ & 0.125653 & 26.57579 & 15.49471 & 0.0007 \\
At most $1^{*}$ & 0.067747 & 9.119681 & 3.841466 & 0.0025 \\
\hline
\end{tabular}

Response to Cholesky One S.D. Innovations 2 S.E.

Response of CPI to $\mathrm{CPI}$
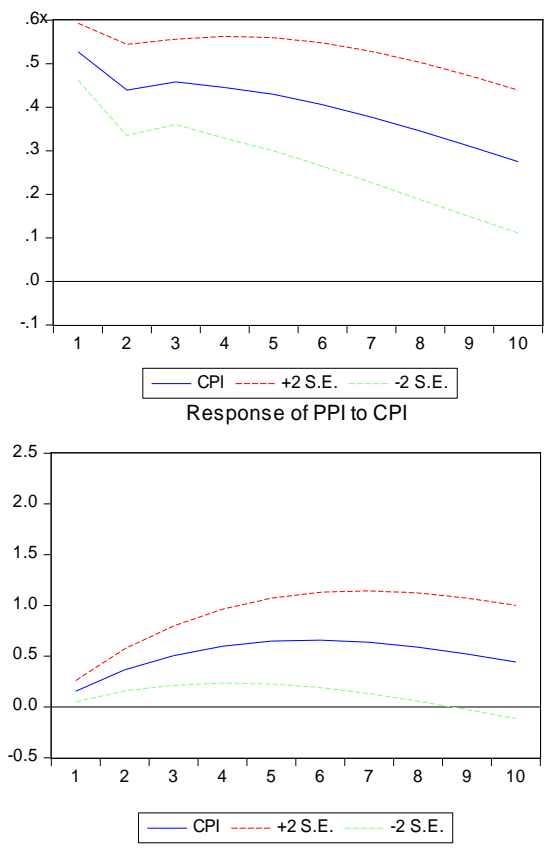

Response of CPI to PPI
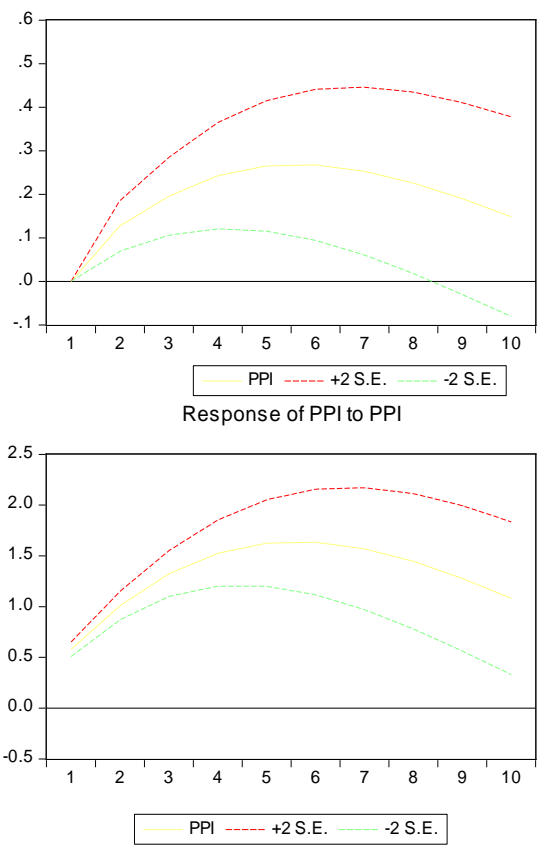

Figure 3. Synthesis of impulse response function.

interference to itself. In the long run, the impact of the consumer price index on itself cannot be ignored. Consumer price index did not respond immediately to the first phase of the producer price index, and began to rise with the extension of the lag period, the sixth period rose to the highest point of 0.27 and then gradually declined. Hence, whether in the long-term or short-term, although the consumer price index will have a certain impact on the producer price index, but the impact is not significant.

The producer price index began to respond to its own impact in the first phase, the fifth period reached a maximum of 1.63, and then began to decline but not to zero, indicating that the producer price index has a certain long-term impact on itself. The producer price index has a certain impact on consumer price index, in the sixth period, it reached a maximum of 0.66 , and then began to slowly decline but not to zero.

To sum up: whether it is the impact of CPI on itself or the impact of PPI on CPI, and reversely, whether it is the impact of PPI on itself or the impact of CPI on PPI, it is always positive. However, this did not affect the long-term deviation between CPI and PPI under the new economic normal. 


\subsection{VEC Model Establishment and Parameter Estimation}

According to Johansen's cointegration test, there is a long-term cointegration relationship between the consumer price index and the producer price index. Based on the previous VAR(2) model, the VEC model can be established to analyze the long-term stability relationship and short-term fluctuations between the two.

The VEC model is as follows:

$$
\begin{aligned}
\left(\begin{array}{l}
\Delta \mathrm{CPI} \\
\Delta \mathrm{PPI}
\end{array}\right)_{t}= & \left(\begin{array}{l}
-0.052804 \\
-0.016248
\end{array}\right)+\left(\begin{array}{cc}
-0.168972 & 0.224526 \\
0.188154 & 0.784060
\end{array}\right)\left(\begin{array}{l}
\Delta C P I \\
\Delta P P I
\end{array}\right)_{t-1} \\
& +\left(\begin{array}{l}
-0.020951 \\
-0.033789
\end{array}\right) \operatorname{vecm}_{t-1}+\left(\begin{array}{l}
\mu_{1 t} \\
\mu_{2 t}
\end{array}\right)
\end{aligned}
$$

where, the cointegration equation $\operatorname{vecm}_{t-1}$ :

$$
\text { vecm }_{t-1}=-195.7560+\mathrm{CPI}_{t-1}+0.923276 \mathrm{PPI}_{t-1}
$$

From the estimation results of the VEC model, the SC value in the model is 1.711131, which indicates that the VEC model is very reasonable. It can be seen from the equation that CPI and PPI of the previous period have a positive effect on the current PPI. For each additional percentage-point increase in CPI of the previous period, the current PPI will increase by 0.188154 percentage-point; for each additional percentage-point increase in PPI of the previous period, the current PPI will increase by 0.784060 percentage-point. The CPI of previous period has a reverse effect on the current CPI, the PPI of previous period has a positive effect on the current CPI. For every one percentage-point increase in the previous period of CPI, the current CPI will decrease by 0.168972 percentage-point; for each additional percentage-point increase in PPI of previous period, the current CPI will increase by 0.224526 percentage points. Vecm is an error correction term, and its coefficient is negative, indicating that the CPI reversely corrects the CPI value of the next period with a value of 0.020951 to reach a long-term equilibrium state, and the PPI reversely corrects the PPI value of the next period with a value of 0.033789 to reach along-term equilibrium state.

Figure 4 is the cointegration curve between the CPI sequence and PPI sequence. From 2008 to the end of 2011, the absolute value of the error correction term deviates greatly, especially in May 2008, August 2009, and August 2011. Short-term fluctuations deviate from long-term equilibrium. After 2012, the fluctuation range of the error correction item began to decrease, and gradually returned to the long-term equilibrium state, which was caused by a series of de-capacity, destocking, and supply-side reforms in China.

\section{Conclusions}

This paper uses the VEC model to empirically analyze consumer price index and producer price index, and studies the cointegration relationship between CPI and PPI, summarizing the following four points:

1) The CPI sequence and the PPI sequence are stabilized after the first-order 


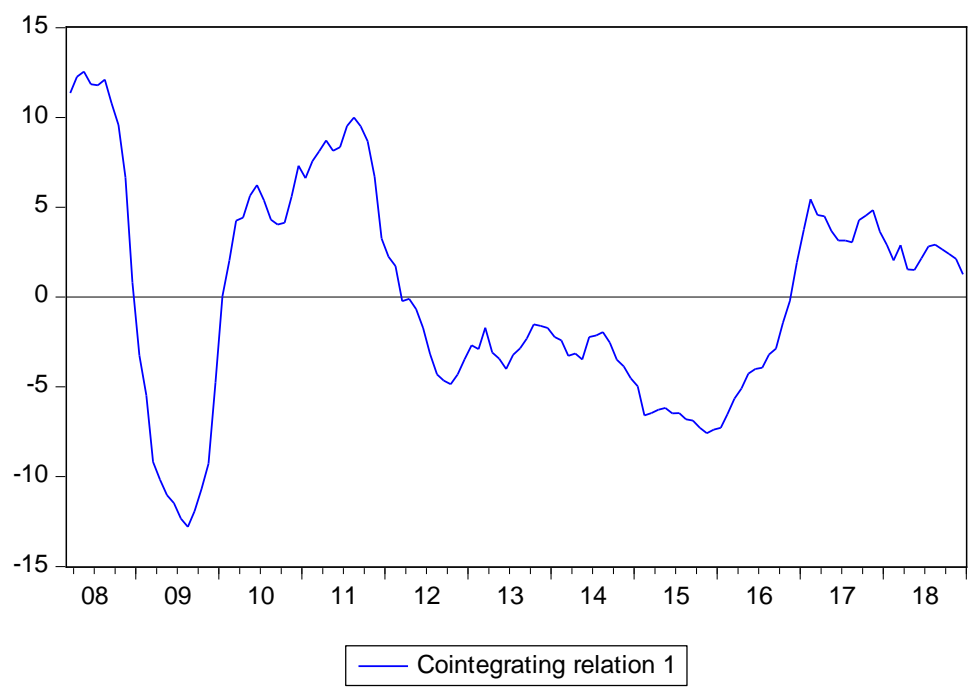

Figure 4. Cointegration diagram of CPI and PPI.

difference, and both belong to the first-order single-order sequence. Using the two-sequence lag phase 2 to be highly significant, the VAR(2) model was constructed, indicating that the current consumer price index and the producer price index will be affected by the changes in the first two periods.

2) There is a cointegration relationship between CPI and PPI, which indicates that there is a long-term stable relationship between consumer price index and producer price index.

3) The impulse response of CPI and PPI indicates that CPI has long-term effects on its own short-term fluctuations about whether it is from short-term fluctuations or long-term effects; CPI has certain impact on PPI, but the impact is not strong. PPI has a great impact on itself and has a long-term impact on CPI, but the impact is not big.

4) The VEC model analyzed the long-term stable relationship between CPI and PPI and the short-term fluctuations. The current CPI will be affected by the reverse impact of the previous CPI and the positive impact of the previous PPI. The current PPI will be affected by the previous CPI, the positive impact and the positive impact of the previous PPI. The coefficient error correction term is a negative value, which plays a role of inversely correcting the next period of CPI and PPI values to achieve a long-term equilibrium state.

\section{Acknowledgements}

Project supported by the National Natural Science Foundation of China (61703117); National Natural Science Foundation of China (61763008); Guangxi Young and Middle-aged Teachers' Basic Ability Improvement Project (2018KY0261).

\section{Conflicts of Interest}

The authors declare no conflicts of interest regarding the publication of this paper. 


\section{References}

[1] Hu, J.M. and Zeng, L.Q. (2017) Economic Stagflation Risk and Its Prevention. Science Press, Beijing.

[2] Shi, K. (2016) Analysis of the VEC Model of CPI and PPI Relationship. Statistics and Decision, No. 3, 83-86.

[3] Chen, Y. (2011) Research on the Relationship between PPI, Enterprise Commodity Price Index, M2 and CPI. Journal of Liaoning University (Philosophy and Social Sciences), 39, 97-103.

[4] He, L.P., Fan, G. and Hu, J.N. (2008) Consumer Price Index and Producer Price Index: Who Drives Who? Economic Research, No. 11, 44-48.

[5] Xu, W.K. (2010) On the Consumer Price Index and Producer Price Index: Who Drives Who? Questioning in a Paper. Economic Research, No. 5, 139-148.

[6] Yang, Z.H., Zhao, Y.L. and Liu, J.H. (2013) Nonlinear Study of CPI and PPI Conduction Mechanisms: Forward Conduction or Reverse Thrust? Economic Research, No. 3, 83-95.

[7] Yang, C. and Chen, L. (2013) Chinese CPI and PPI: Causality and Transmission Mechanism. Journal of Xiamen University (Philosophy and Social Sciences), No. 3, 1-9.

[8] Sims, C.A. (1980) Macroeconomics and Reality. Econometrica, 48, 1-48.

[9] Johansen, S. and Juselius, K. (1990) Maximum Likelihood Estimation and Inferences on Cointegration-With Applications to the Demand for Money. Oxford Bulletin of Economics and Statistics, 52, 169-210.

[10] Gao, T.M. (2016) Econometric Analysis Methods and Modeling-EViews Applications and Examples. 3rd Edition, Tsinghua University Press, Beijing. 